\title{
A few new (?) facts about infinite elements
}

\author{
L. Demkowicz ${ }^{\mathrm{a}, *}$, Jie Shen ${ }^{\mathrm{b}}$ \\ ${ }^{a}$ Institute for Computational Engineering and Sciences, The University of Texas at Austin, \\ 201 E. 24th St. ACE 4.102, Austin, TX 78712, USA \\ ${ }^{\mathrm{b}}$ Department of Mathematics, Purdue University, USA
}

Received 9 December 2004; received in revised form 2 March 2005; accepted 10 April 2005

\begin{abstract}
We discuss the (conjugated) Bubnov-Galerkin and Petrov-Galerkin infinite element (IE) discretizations to Helmholtz equation including the use of elements of locally variable order, optimal choice of IE shape functions, calculation of Echo Area (EA), and automatic $h p$-adaptivity. The discussion is illustrated with 2D numerical experiments.
\end{abstract}

(C) 2005 Elsevier B.V. All rights reserved.

MSC classification: $65 \mathrm{~N} 30 ; 35 \mathrm{~L} 15$

Keywords: Helmholtz equation; Infinite element; $h p$ finite elements; Echo Area

\section{Introduction}

The idea of coupled finite element (FE)/infinite element (IE) approximations for exterior wave propagation problems dates back to the pioneering contributions of Bettess, and Bettess and Zienkiewicz, see [5] and the literature cited therein.

The works of Astley et al. [3], Cremers et al. [8], Givoli [13] and many others recognized the spectral character of the approximation and pointed to the necessity of multipole expansions. Burnett [7] revolutionized the approach from the practical point of view, by introducing a new, symmetric unconjugated formulation, and using prolate and oblate spheroidal elements, we refer to [18] for a recent review on the subject.

Contrary to the concept of Perfectly Matched Layer [4], and other techniques based on Absorbing Boundary Conditions (ABC's), the conjugated element of Astley et al. [3], aims at obtaining the solution in the whole unbounded domain.

\footnotetext{
* Corresponding author. Tel.: +1 512471 3312; fax: +1 5124718694.

E-mail address: leszek@ices.utexas.edu (L. Demkowicz).
} 
For Laplace equation (coercive problems in general), convergence analysis for coupled FE/IE discretizations reduces to the proof of approximability, see [12]. With the stability granted, the IE discretization in spherical or ellipsoidal coordinates, can be replaced with techniques better suited for more complex geometries, and adaptivity, see e.g. [6] for the idea of inverted finite elements. The energy $\left(L^{2}\right.$ norm of the solution gradient) is finite which allows for a standard Bubnov-Galerkin discretization. It is perhaps worth mentioning that, for unbounded domains, $L^{2}$-integrability of derivatives does not imply the $L^{2}$-integrability of the function, solution of the Laplace exterior problem "lives" in a weighted $L^{2}$ space only.

The Helmholtz equation is dramatically different. The energy of the solution is no longer finite. This is not a surprise; solution to the Helmholtz equation is the steady-state solution corresponding to a time-harmonic forcing term and infinite time. For each finite time interval, the energy "pumped" into the system is finite but, over the infinite time, becomes infinite. This prohibits the use of identical trial and test functions in the standard Bubnov-Galerkin method, if the energy and mass terms in the variational formulation are to be Lebesgue integrable. The Petrov-Galerkin formulation of Astley et al. [3] becomes the natural choice.

The unconjugated Bubnov-Galerkin symmetric formulation of Burnett is possible due to the interpretation of the integrals in the Cauchy Principal Value (CPV) sense. However, it appears that the possibility of using the same idea for the conjugated element has been unexplored.

Convergence analysis for the exterior Helmholtz problem is much more challenging than for the Laplace equation. Due to the unboundedness of the domain, the compact perturbation argument does not work. One possibility of analyzing the convergence is to give up on the analysis in the whole exterior domain, and treat the infinite element as an implicit ABC. The exact Dirichlet-to-Neumann (DtN) operator is then replaced with its approximate IE counterpart. This was the idea behind the analysis presented in [10] where sufficient conditions for convergence, expressed in terms of eigenvalues of the approximate DtN operator, were formulated. ${ }^{1}$ Those eigenvalues were determined only numerically and, in a strict mathematical sense, the proof of convergence for the infinite elements remains an open problem.

Spectral discretizations are much more sensitive to conditioning problems, and infinite elements of higher order are not exception. Conditioning problems have been reported by many authors, see e.g. $[2,16]$. Similar problems have been encountered in context of using infinite elements for solving exterior Maxwell problems and determining Radar Cross Sections (RCS) [19].

This short contribution has been motivated with the idea of the second author who, several years ago, suggested to employ rational functions generated from the (integrated) Jacobi polynomials for infinite element shape functions, as a possible means for overcoming the conditioning problems. The results reported in this work have been obtained in context of a larger effort aimed at using coupled FE/IE $h p$ discretizations for determining the far-field patterns represented by Echo Area (EA) in acoustics, and Radar Cross Section (RCS) in electromagnetics.

In process of putting together a chapter on $h p$-adaptivity for exterior boundary value problems, the first author has learned a few extra new (at least for him) facts that (we hope) may be useful to the IE community. Those include the following issues:

- The possibility of a Bubnov-Galerkin discretization for the conjugated element.

- The possibility of a simultaneous construction of IE shape functions for both 2D and 3D problems.

- The possibility of varying locally the IE spectral order of approximation.

- The possibility of a direct evaluation of Echo Area from the IE representation, without any costly postprocessing.

\footnotetext{
${ }^{1}$ Only the case of spherical infinite elements was analyzed.
} 
All derivations have been done for cylindrical (spherical) truncating surfaces only, and we report here only two-dimensional calculations.

\section{Formulation of the problem}

We shall consider the exterior boundary value problem for the Helmholtz equation, representing a class of acoustical rigid scattering problems.

Let $\Omega_{\text {int }}$ be an open bounded domain in $\mathbb{R}^{n}$, we set $\Omega=\mathbb{R}^{n}-\bar{\Omega}_{\text {int }}$. Given an incident pressure wave $u^{\text {inc }}$ that satisfies the Helmholtz equation in the whole space

$$
-\Delta u^{\text {inc }}-k^{2} u^{\text {inc }}=0 \text { in } \mathbb{R}^{n},
$$

we look for a scattered pressure wave $u$ that satisfies:

- The same Helmholtz equation, but only in the exterior domain

$$
-\Delta u-k^{2} u=0 \quad \text { in } \Omega=\mathbb{R}^{n}-\Omega_{\text {int }},
$$

- Neumann boundary condition on boundary $\Gamma=\partial \Omega=\partial \Omega_{\text {int }}$

$$
\frac{\partial\left(u+u^{\text {inc }}\right)}{\partial n}=0
$$

- Sommerfeld radiation condition at infinity

$$
\frac{\partial u}{\partial r}+\mathrm{i} k u \in L^{2}(\Omega)
$$

Here $k=\frac{\omega}{c}>0$ is the wave number with $\omega$ representing the angular frequency, and $c$ the speed of sound in the (homogeneous) medium, $\boldsymbol{n}$ is the outward unit vector, and $r$ stands for the radial coordinate corresponding to polar coordinates with the origin at an arbitrary point, typically chosen inside of the interior domain $\Omega_{\text {int }}$ occupied by the rigid obstacle (scatterer). As the gradient of the pressure is proportional to the velocity vector, the Neumann boundary condition corresponds to the requirement that the normal component of the total velocity vector must vanish on the boundary of the rigid obstacle. The sum of the incident and scattered pressures represents the total pressure wave. In practice, the incident pressure wave corresponds to a source located away from the obstacle. If the location of the source is far enough, the incident pressure wave may be assumed in the form of a plane wave (source at infinity)

$$
u^{\text {inc }}(\boldsymbol{x})=u_{0} \mathrm{e}^{-\mathrm{i} k e \cdot \boldsymbol{x}},
$$

where $u_{0}$ is a scaling factor, and unit vector $\boldsymbol{e}$ represents the direction of the incoming incident wave. As the problem is linear, we can assume $u_{0}=1$. The role of the incident wave is therefore to provide only a driving Neumann load data on boundary $\Gamma$

$$
\frac{\partial u}{\partial n}=g:=-\frac{\partial u^{\mathrm{inc}}}{\partial n}
$$

The Sommerfeld radiation condition represents a requirement that the scattered wave must be outgoing to infinity, and it can be represented in many different but equivalent forms. The form used here has a particular advantage of being independent of space dimension. Notice that the formula for the plane wave, and the sign in the Sommerfeld radiation condition correspond to the $\mathrm{e}^{\mathrm{i} \omega t}$ ansatz in time. 
Of particular interest to us, will be the computation of the monostatic Echo Area (EA) defined as

$$
\lim _{r \rightarrow \infty}|u(r \hat{\boldsymbol{x}})| r^{\alpha} .
$$

Here $\hat{\boldsymbol{x}}$ is a point on the unit sphere (circle) opposite to the direction of the incident wave, $\boldsymbol{e}=-\hat{\boldsymbol{x}}$, and $u$ is the corresponding scattered wave. Coefficient

$$
\alpha= \begin{cases}\frac{1}{2} & \text { in } \mathbb{R}^{2}, \\ 1 & \text { in } \mathbb{R}^{3},\end{cases}
$$

reflects the decay rate of the leading term (far-field pattern) of the solution.

\section{Infinite element discretization}

The idea of coupled finite/infinite elements is illustrated in Fig. 1. The scatterer is surrounded with an ellipsoidal truncating surface partitioning the exterior domain $\Omega$ into the "near-field" domain $\Omega^{a}$ in between the scatterer and the truncating surface, and the remaining "far-field" domain $\Omega_{a}$. The near-field domain is then meshed with finite elements, and the "far-field" domain is discretized with infinite elements, in a fully conforming way. In practice, the exact geometry of the truncating surface is replaced with its FE isoparametric approximation. The infinite elements are aligned with the coordinate lines of the ellipsoidal system of coordinates. In our discussion, we will restrict ourselves to the simplest case of cylindrical (2D) or spherical (3D) truncating surface of radius $a$, only.

\subsection{Variational formulation}

We will discuss the variational formulation for the "far-field" part of the domain first.

We introduce a larger circle (sphere in 3D) of radius $R$, and follow the standard derivation of the variational formulation for the truncated far-field domain

$$
\Omega_{a}^{R}=\{\boldsymbol{x}: a<|\boldsymbol{x}|<R\} .
$$

Assuming $u, v \in H^{1}\left(\Omega_{a}^{R}\right)$, we obtain, ${ }^{2}$

$$
\int_{\Omega_{a}^{R}}\left\{\nabla u \nabla \bar{v}-k^{2} u \bar{v}\right\} \mathrm{d} \boldsymbol{x}+\mathrm{i} k \int_{S_{R}} u \bar{v} \mathrm{~d} s=\int_{S_{a}} \frac{\partial u}{\partial n} \bar{v} \mathrm{~d} s+\int_{S_{R}} w \bar{v} \mathrm{~d} s, \quad \forall v .
$$

Here $w:=\frac{\partial u}{\partial r}+\mathrm{i} k u$, according to the Sommerfeld condition, denotes an unknown function, $L^{2}$-integrable in $\Omega^{a}$, and $S_{a}$ is the truncating circle or sphere.

We want to pass with $R \rightarrow+\infty$ in (3.4). The $L^{2}$-integrability of function $w$ implies that the unknown term involving function $w$ will vanish. The first boundary integral on the right-hand side is independent of $R$, so we only need to focus on the two integrals on the left-hand side only.

\subsubsection{System of coordinates}

We will use a curvilinear system of coordinates, corresponding to an arbitrary parametrization of the truncating surface. In $2 \mathrm{D}$, we have

$$
\boldsymbol{x}(r, \xi)=r \boldsymbol{x}_{a}(\xi), \quad r>1, \quad\left|\boldsymbol{x}_{a}\right|=a,
$$

where $\boldsymbol{x}_{a}(\xi)$ denotes a parametrization of the truncating circle of radius $a$.

\footnotetext{
${ }^{2} \bar{v}$ represents the complex conjugate of $v$.
} 


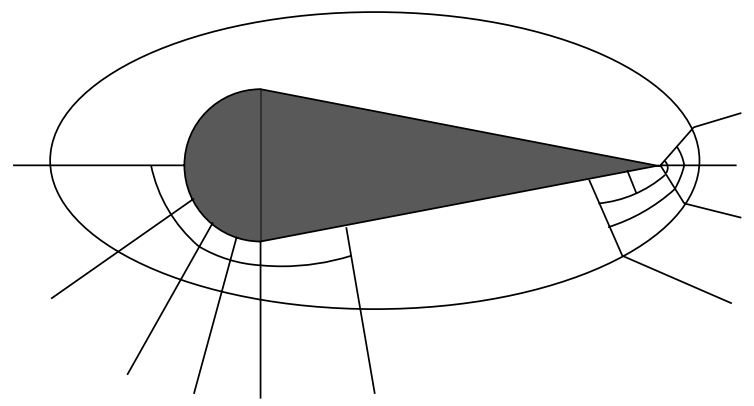

Fig. 1. FE/IE mesh around a scatterer.

We have the standard formulas

$$
\begin{aligned}
& \frac{\partial \boldsymbol{x}}{\partial r}=\boldsymbol{x}_{a}=a \boldsymbol{e}_{r}, \quad \text { where } \boldsymbol{e}_{r}:=a^{-1} \boldsymbol{x}_{a}, \\
& \frac{\partial \boldsymbol{x}}{\partial \xi}=r \frac{\mathrm{d} \boldsymbol{x}_{a}}{\mathrm{~d} \xi}=r\left|\frac{\mathrm{d} \boldsymbol{x}_{a}}{\mathrm{~d} \xi}\right| \boldsymbol{e}, \quad \text { where } \boldsymbol{e}:=\left|\frac{\partial \boldsymbol{x}}{\partial \xi}\right|^{-1} \frac{\partial \boldsymbol{x}}{\partial \xi}, \\
& \nabla u=\frac{1}{a} \frac{\partial u}{\partial r} \boldsymbol{e}_{r}+\frac{1}{r} \underbrace{\frac{\partial u}{\partial \xi}\left|\frac{\mathrm{d} \boldsymbol{x}_{a}}{\mathrm{~d} \xi}\right|^{-1} \boldsymbol{e}}_{\nabla_{S_{a}}}=\frac{1}{a} \frac{\partial u}{\partial r} \boldsymbol{e}_{r}+\frac{1}{r} \nabla_{S_{a}} u,
\end{aligned}
$$

with $\nabla_{S_{a}}$ denoting the gradient on the circle $S_{a}$.

The corresponding formulas in $3 \mathrm{D}$ are slightly more complex. The system of coordinates is defined as

$$
\boldsymbol{x}\left(r, \xi_{\beta}\right)=r \boldsymbol{x}_{a}\left(\xi_{\beta}\right), \quad \beta=1,2, \quad\left|\boldsymbol{x}_{a}\left(\xi_{\beta}\right)\right|=a, \quad r>1,
$$

where $\boldsymbol{x}_{a}\left(\xi_{\beta}\right)$ is a parametrization of the truncating sphere of radius $a$. The basis vectors are

$$
\boldsymbol{a}_{\beta}=r \frac{\partial \boldsymbol{x}_{a}}{\partial \xi_{\beta}}=r\left|\frac{\partial \boldsymbol{x}_{a}}{\partial \xi_{\beta}}\right| \boldsymbol{e}_{\beta}, \quad \boldsymbol{a}_{r}=\boldsymbol{x}_{a}=a \boldsymbol{e}_{r} .
$$

We assume that the parameters $\beta$ have been ordered in such a way that $\boldsymbol{e}_{r}=\boldsymbol{e}_{1} \times \boldsymbol{e}_{2}$. The cobasis vectors are given by

$$
\boldsymbol{a}^{1}=r^{-1} \mathrm{jac}_{a}^{-1}\left(\frac{\partial \boldsymbol{x}_{a}}{\partial \xi_{2}} \times \boldsymbol{e}_{r}\right), \quad \boldsymbol{a}^{1}=-r^{-1} \mathrm{jac}_{a}^{-1}\left(\frac{\partial \boldsymbol{x}_{a}}{\partial \xi_{1}} \times \boldsymbol{e}_{r}\right), \quad \boldsymbol{a}^{r}=a^{-1} \boldsymbol{e}_{r},
$$

where

$$
\mathrm{jac}_{a}=\left(\frac{\partial \boldsymbol{x}_{a}}{\partial \xi_{1}} \times \frac{\partial \boldsymbol{x}_{a}}{\partial \xi_{2}}\right) \cdot \boldsymbol{e}_{r}
$$

Denoting by $\hat{\boldsymbol{a}}_{\beta}, \hat{\boldsymbol{a}}^{\beta}$ basis and cobasis vectors on the truncating surface, i.e.

$$
\boldsymbol{a}_{\beta}=r \hat{\boldsymbol{a}}_{\beta}, \quad \boldsymbol{a}^{\beta}=r^{-1} \hat{\boldsymbol{a}}^{\beta},
$$

we obtain a formula for the gradient similar to its $2 \mathrm{D}$ version $(3.5)_{3}$

$$
\boldsymbol{\nabla} u=r^{-1} \underbrace{\frac{\partial u}{\partial \xi_{\beta}} \hat{\boldsymbol{a}}^{\beta}}_{\nabla_{S_{a}} u}+a^{-1} \frac{\partial u}{\partial r} \boldsymbol{e}_{r} .
$$


As usual, repeated indices indicate summation.

The sesquilinear form in the variational formulation over domain $\Omega_{a}^{R}$ is computed as follows:

$$
\begin{aligned}
b(u, v)= & a^{-1} \int_{S_{a}} \int_{1}^{\rho}\left(\frac{\partial u}{\partial r} \frac{\partial \bar{v}}{\partial r}-(k a)^{2} u \bar{v}\right) r^{2 \alpha} \mathrm{d} r \mathrm{~d} S_{a}+a \int_{S_{a}} \int_{1}^{\rho} \frac{1}{r^{2}} \nabla_{S_{a}} u \nabla_{S_{a}} \bar{v} r^{2 \alpha} \mathrm{d} r \mathrm{~d} S_{a} \\
& +\mathrm{i} k \rho^{2 \alpha} \int_{S_{a}} u(\rho, \cdot) \bar{v}(\rho, \cdot) \mathrm{d} S_{a},
\end{aligned}
$$

where $\rho a=R, \mathrm{~d} S_{a}=\left|\frac{\mathrm{d} x_{a}}{\mathrm{~d} \xi}\right| \mathrm{d} \xi$ in $2 \mathrm{D}$, and $\mathrm{d} S_{a}=\mathrm{jac}_{a} \mathrm{~d} \xi_{1} \mathrm{~d} \xi_{2}$ in $3 \mathrm{D}$.

\subsubsection{Incorporating the far-field pattern: change of dependent variable}

Consistent with the far-field pattern, known from the Atkinson-Wilcox expansion, we shall seek the solution $u$ in the form

$$
u=\frac{\mathrm{e}^{-\mathrm{i} k a(r-1)}}{r^{\alpha}} U
$$

where $U$ is the new unknown, and $\alpha$ is a dimension dependent constant defined in (2.3). An identical substitution will be used for test functions. The idea is to substitute these representations into the variational formulation over the truncated far-field domain $\Omega_{a}^{R}$, cancel out all Lebesgue non-integrable terms, and then pass with $R \rightarrow \infty$.

Substituting (3.13) into the second integral in the formula for the sesquilinear form (3.12) yields simply

$$
a \int_{S_{a}} \int_{1}^{\rho} \frac{1}{r^{2}} \nabla_{S_{a}} U \nabla_{S_{a}} \bar{V} \mathrm{~d} r \mathrm{~d} S_{a}
$$

and we need to focus only on the inner integral of the sum of the first and the third term

$$
a^{-1} \int_{1}^{\rho}\left(\frac{\partial u}{\partial r} \frac{\partial \bar{v}}{\partial r}-(k a)^{2} u \bar{v}\right) r^{2 \alpha} \mathrm{d} r+\mathrm{i} k \rho^{2 \alpha} u(\rho) \bar{v}(\rho)
$$

for a particular value of $\xi$.

We shall proceed with the substitution (3.13) in three steps.

Step 1: Taking out the oscillating factor. By setting

$$
u(r)=\mathrm{e}^{-\mathrm{i} k a(r-1)} U(r), \quad \bar{v}(r)=\mathrm{e}^{\mathrm{i} k a(r-1)} \bar{V}(r),
$$

we obtain

$$
\frac{\partial u}{\partial r}=\left(\frac{\partial U}{\partial r}-\mathrm{i} k a U\right) \mathrm{e}^{-\mathrm{i} k a(r-1)}, \quad \frac{\partial \bar{v}}{\partial r}=\left(\frac{\partial \bar{V}}{\partial r}+\mathrm{i} k a \bar{V}\right) \mathrm{e}^{\mathrm{i} k a(r-1)}
$$

This leads to the following modification of the integrand (3.15):

$$
a^{-1} \int_{1}^{\rho}\left[\frac{\partial U}{\partial r} \frac{\partial \bar{V}}{\partial r}+\mathrm{i} k a\left(\frac{\partial U}{\partial r} \bar{V}-U \frac{\partial \bar{V}}{\partial r}\right)\right] r^{2 \alpha} \mathrm{d} r+\mathrm{i} k \rho^{2 \alpha} U(\rho) \bar{V}(\rho) .
$$

Notice that the cancellation obtained so far, does not guarantee Lebesgue integrability of all the remaining terms. With a leading term $1 / r$ for both $U$ and $V$, the first term in the integrand is integrable but the second and third are not. In order to avoid introducing new symbols, we shall trade now symbols $U, V$ back for $u, v$

$$
a^{-1} \int_{1}^{\rho}\left[\frac{\partial u}{\partial r} \frac{\partial \bar{v}}{\partial r}+\mathrm{i} k a\left(\frac{\partial u}{\partial r} \bar{v}-u \frac{\partial \bar{v}}{\partial r}\right)\right] r^{2 \alpha} \mathrm{d} r+\mathrm{i} k \rho^{2 \alpha} u(\rho) \bar{v}(\rho) .
$$


Step 2: Taking out the Jacobian. We substitute

$$
\begin{aligned}
& u=r^{-\alpha} U, \quad \bar{v}=r^{-\alpha} \bar{V}, \\
& \frac{\partial u}{\partial r}=\left(\frac{\partial U}{\partial r}-\frac{\alpha}{r} U\right) r^{-\alpha}, \quad \frac{\partial \bar{v}}{\partial r}=\left(\frac{\partial \bar{V}}{\partial r}-\frac{\alpha}{r} \bar{V}\right) r^{-\alpha} .
\end{aligned}
$$

This eliminates the Jacobian from the integrand (3.17)

$$
a^{-1} \int_{1}^{\rho}\left[\left(\frac{\partial U}{\partial r}-\frac{\alpha}{r} U\right)\left(\frac{\partial \bar{V}}{\partial r}-\frac{\alpha}{r} \bar{V}\right)+\mathrm{i} k a\left(\frac{\partial U}{\partial r} \bar{V}-U \frac{\partial \bar{V}}{\partial r}\right)\right] \mathrm{d} r+\mathrm{i} k U(\rho) \bar{V}(\rho) .
$$

Integration by parts of the last term in the integrand leads to

$$
a^{-1} \int_{1}^{\rho}\left[\left(\frac{\partial U}{\partial r}-\frac{\alpha}{r} U\right)\left(\frac{\partial \bar{V}}{\partial r}-\frac{\alpha}{r} \bar{V}\right)+2 \mathrm{i} k a \frac{\partial U}{\partial r} \bar{V}\right] \mathrm{d} r+\mathrm{i} k U(1) \bar{V}(1) .
$$

Expanding the first term leads to

$$
a^{-1} \int_{1}^{\rho}\left[\frac{\partial U}{\partial r} \frac{\partial \bar{V}}{\partial r}-\frac{\alpha}{r} \frac{\partial U}{\partial r} \bar{V}-\frac{\alpha}{r} U \frac{\partial \bar{V}}{\partial r}+\frac{\alpha^{2}}{r^{2}} U \bar{V}+2 \mathrm{i} k a \frac{\partial U}{\partial r} \bar{V}\right] \mathrm{d} r+\mathrm{i} k U(1) \bar{V}(1) .
$$

Finally, after integrating by parts the third term, we get

$$
a^{-1} \int_{1}^{\rho}\left[\frac{\partial U}{\partial r} \frac{\partial \bar{V}}{\partial r}+\frac{\alpha(\alpha-1)}{r^{2}} U \bar{V}+2 \mathrm{i} k a \frac{\partial U}{\partial r} \bar{V}\right] \mathrm{d} r+\frac{\alpha+\mathrm{i} k a}{a} U(1) \bar{V}(1)-\frac{\alpha}{\rho a} U(\rho) \bar{V}(\rho) .
$$

Upon passing with $\rho \rightarrow \infty$, we obtain

$$
a^{-1} \int_{1}^{\infty}\left[\frac{\partial U}{\partial r} \frac{\partial \bar{V}}{\partial r}+\frac{\alpha(\alpha-1)}{r^{2}} U \bar{V}+2 \mathrm{i} k a \frac{\partial U}{\partial r} \bar{V}\right] \mathrm{d} r+\frac{\alpha+\mathrm{i} k a}{a} U(1) \bar{V}(1) .
$$

Step 3: Change of the independent variable. Our final substitution involves mapping a neighborhood of infinity into a neighborhood of zero. We substitute

$$
r=\frac{1}{x}, \quad \mathrm{~d} r=-\frac{1}{x^{2}} \mathrm{~d} x, \quad \frac{\mathrm{d} U}{\mathrm{~d} r}=-x^{2} \frac{\mathrm{d} U}{\mathrm{~d} x} .
$$

The final formula for the sesquilinear form is

$$
\begin{aligned}
b(u, v)= & \int_{S_{a}} \int_{0}^{1}\left\{a^{-1}\left[x^{2} \frac{\partial U}{\partial x} \frac{\partial \bar{V}}{\partial x}+\alpha(\alpha-1) U \bar{V}-2 \mathrm{i} k a \frac{\partial U}{\partial x} \bar{V}\right]+a \nabla_{S_{a}} U \nabla_{S_{a}} \bar{V}\right\} \mathrm{d} x \mathrm{~d} S_{a} \\
& +\frac{\alpha+\mathrm{i} k a}{a} \int_{S_{a}} U(1, \cdot) \bar{V}(1, \cdot) \mathrm{d} S_{a}
\end{aligned}
$$

where the combined transformation is

$$
u(r, \xi)=\frac{\mathrm{e}^{-\mathrm{i} k a(r-1)}}{r^{\alpha}} U(r, \xi)=\mathrm{e}^{-\mathrm{i} k a\left(x^{-1}-1\right)} x^{\alpha} U(x, \xi),
$$

with an identical formula for the test functions.

The ultimate variational formulation is obtained by taking the standard variational formulation for the "near-field" domain

$$
\int_{\Omega^{a}}\left\{\nabla u \nabla \bar{v}-k^{2} u \bar{v}\right\} \mathrm{d} \boldsymbol{x}=\int_{\Gamma} g \bar{v} \mathrm{~d} s+\int_{S_{a}} \frac{\partial u}{\partial n} \bar{v} \mathrm{~d} s, \quad \forall v
$$


and summing it up with (3.4). We obtain the final variational formulation in the standard abstract form: Find $u \in X$ such that

$$
b(u, v)=l(v), \quad \forall v \in Y,
$$

where $X$ and $Y$ are some appropriate functional spaces, and the sesquilinear and antilinear forms are given by

$$
\begin{aligned}
b(u, v)= & \int_{\Omega^{a}}\left\{\nabla u \nabla \bar{v}-k^{2} u \bar{v}\right\} \mathrm{d} \boldsymbol{x} \\
= & \int_{S_{a}} \int_{0}^{1}\left\{a^{-1}\left[x^{2} \frac{\partial U}{\partial x} \frac{\partial \bar{V}}{\partial x}+\alpha(\alpha-1) U \bar{V}-2 \mathrm{i} k a \frac{\partial U}{\partial x} \bar{V}\right]+a \nabla_{S_{a}} U \nabla_{S_{a}} \bar{V}\right\} \mathrm{d} x \mathrm{~d} S_{a} \\
& \quad+\frac{\alpha+\mathrm{i} k a}{a} \int_{S_{a}} U(1, \cdot) \bar{V}(1, \cdot) \mathrm{d} S_{a} \\
l(v)= & \int_{\Gamma} g \bar{v} \mathrm{~d} s .
\end{aligned}
$$

At this point, the variational formulation is purely formal. Finding the right functional setting for the case of identical trial and test spaces $X=Y$, remains an open problem.

\subsection{Selecting IE radial shape functions}

As usual, shape functions for the infinite elements are constructed by taking tensor products of onedimensional radial shape functions of coordinate $x$ (or, equivalently, $r$ ) and standard FE shape functions corresponding to the edge (face in 3D) lying on the truncating circle (sphere)

$$
U(x, \xi)=\sum_{k l} U_{k l} \psi_{k}(x) e_{l}(\xi)
$$

The corresponding infinite element space of test functions is the tensor product of polynomials of order $N$ in $x$ and polynomials of order $p$ in $\xi, \mathscr{P}^{N} \otimes \mathscr{P}^{p}$. Here $N$ is the (radial) order of the infinite element. The construction extends in a standard way to elements of variable order.

There are many possible choices for the IE radial shape functions.

\subsubsection{Same trial and test functions (Bubnov-Galerkin)}

The leading term (derivative times derivative) in (3.24) suggests the use of integrated Jacobi polynomials $P_{n}^{(0,2)}$ (transformed to interval $\left.(0,1)\right)$

$$
\psi_{j}(\xi)= \begin{cases}1, & j=0 \\ \int_{\xi}^{1} P_{j-1}^{(0,2)}(2 t-1) \mathrm{d} t, & j \geqslant 1 .\end{cases}
$$

Only the first IE shape functions "connects" with the adjacent finite element, the remaining ones vanish on the truncating surface and, in terminology of $h p$ methods, they can be classified as IE "bubbles". Shape functions $\psi_{j}, j \geqslant 1$, can be normalized to satisfy a desired scaling condition.

The same shape functions are used for the test functions. Recall that Jacobi polynomials $P_{n}^{(0,2)}$ are orthogonal in the weighted $L^{2}$ inner product

$$
\int_{-1}^{1}(1+t)^{2} u^{\prime} v^{\prime} \mathrm{d} t
$$

After rescaling $x=2 t-1$, the corresponding polynomials, defined on the unit interval $(0,1)$, are orthogonal in the inner product 


$$
\int_{0}^{1} x^{2} u^{\prime} v^{\prime} \mathrm{d} x
$$

corresponding to the first term in the sesquilinear form (3.24). The rationale of the choice is that, asymptotically in order $N$, the leading term is most responsible for conditioning problems.

Notice that, except for the choice of the radial shape functions, the representation for the approximate solution is standard

$$
u(r, \xi)=\frac{\mathrm{e}^{-\mathrm{i} k a(r-1)}}{r^{\alpha}} \sum_{j} \underbrace{\sum_{l} U_{j l} e_{l}(\xi)}_{u_{j}(\xi)} \psi_{j}\left(\frac{1}{r}\right) .
$$

The main point is the choice of the same test functions, possible only because of the interpretation of the integrals in the CPV sense. Notice also that the construction is identical for both 2D and 3D problems. The difference between 2D and 3D is reflected only by different values of coefficient $\alpha$ in formulas (3.25) and (3.24).

We emphasize, however, that even with the same trial and test functions, the corresponding stiffness matrix is neither hermitian nor complex-symmetric. Consequently, a general complex solver must be used.

\subsubsection{Different trial and test functions (Petrov-Galerkin)}

If all the integrals are to be interpreted in the Lebesgue sense only, the leading term for the test functions $V(x)$ must be $x^{2 \alpha}$. In other words, the test functions must be of order $1 / r^{2}$ in $2 \mathrm{D}$, and order $1 / r^{3}$ in $3 \mathrm{D}$. Replacing $V$ with $x^{2 \alpha} V$ in (3.24), we obtain a modified formula for the sesquilinear form

$$
\begin{aligned}
b(u, v)= & \int_{S_{a}} \int_{0}^{1}\left\{a^{-1}\left[x^{2+2 \alpha} \frac{\partial U}{\partial x} \frac{\partial \bar{V}}{\partial x}+\alpha(\alpha-1) x^{2 \alpha} U \bar{V}+\left(2 \alpha x^{2 \alpha-1}-2 \mathrm{i} k a\right) x^{2 \alpha} \frac{\partial U}{\partial x} \bar{V}\right]\right. \\
& \left.+a x^{2 \alpha} \nabla_{S_{a}} U \nabla_{S_{a}} \bar{V}\right\} \mathrm{d} x \mathrm{~d} S_{a}+\frac{\alpha+\mathrm{i} k a}{a} \int_{S_{a}} U(1, \cdot) \bar{V}(1, \cdot) \mathrm{d} S_{a} .
\end{aligned}
$$

The leading term suggests now the use of integrated Jacobi polynomials $P_{n}^{(0,3)}$ in $2 \mathrm{D}$, and integrated Jacobi polynomials $P_{n}^{(0,4)}$ in $3 \mathrm{D}$. The choice is no longer dimension independent. In $2 \mathrm{D}$, we have

$$
\psi_{j}(\xi)= \begin{cases}1, & j=0, \\ \int_{\xi}^{1} P_{j-1}^{(0,3)}(2 t-1) \mathrm{d} t, & j \geqslant 1 .\end{cases}
$$

Notice that the substitution $V:=x^{2 \alpha} V$ is equivalent with the choice of different test shape functions

$$
\phi_{j}(x)=x^{2 \alpha} \psi_{j}(x) \text {. }
$$

It is for this reason that we identify it as the Petrov-Galerkin method. With such a choice of test functions, there is no need for the CPV interpretation of the integrals. In terms of spaces, the method is identical with the element of Astley et al. [3]. We will compare the two methods with simple 2D numerical experiments in the next section.

\section{Numerical experiments}

\subsection{Implementation details}

The infinite element was implemented within $2 D h p 90$, a two-dimensional code supporting automatic $h p$ adaptivity for both $H^{1}$ - and $H$ (curl)-conforming hybrid meshes consisting of isoparametric quads and triangles [9]. Shape functions $e_{l}(\xi)$ in (3.28) have to match the FE shape functions used in the code and, in all 
reported experiments, we have used the standard choice - integrated Legendre polynomials. Integration of infinite element stiffness matrix was done using the standard Gauss-Legendre quadrature, with the number of integration points equal to $p+1$ in the tangential, and $N+1$ in the radial directions, where $p$ and $N$ denote the order of the infinite element in the tangential and the radial directions, respectively.

\subsubsection{Automatic hp-adaptivity}

We refer to [11] for details on the algorithm executing the automatic $h p$-adaptivity. Starting with an initial coarse mesh, we refine the mesh globally in both $h$ and $p$, and solve the problem on the fine mesh. The next optimal coarse mesh is obtained then by minimizing the projection-based interpolation error of the fine grid solution with respect to the coarse grid. More precisely, the optimal coarse mesh is obtained by maximizing the rate with which the interpolation error decreases, as the current coarse grid undergoes selective, local $h p$-refinements. One of the primary goals of the presented research is to determine whether the strategy can be used for the scattering problems. The mesh optimization is restricted to the near-field (truncated) domain only, i.e. the infinite elements are treated as an implicit implementation of ABC's of arbitrary order.

\subsubsection{Choice of radial order $N$}

The infinite elements in the initial mesh are assumed to be isotropic, i.e. order $N$ in the radial direction is set to the corresponding order $p$ of the edge on the truncating circle. We always begin with elements of second order.

During the $h p$-refinements, edges on the truncating circle get $p$ - or $h$-refined. Every time, the edge is $p$ refined, its IE radial order is updated, i.e. $N$ is increased to $N+1$. We also increase the IE order when the edge is $h$-refined. Therefore, in presence of $h$-refinements, we encounter infinite elements with radial order $N$ greater than the FE order $p$. This reflects the philosophy that any improvement in the approximation properties on the truncating circle, should be accompanied with the corresponding improvement in the radial direction as well.

In the presented experiments (due to software related limitations), the IE radial order has been restricted to $N \leqslant 9$.

\subsection{Calculation of Echo Area}

\subsubsection{Direct evaluation using the IE solution}

The simplest way to evaluate EA is based on using the shape functions (3.28). More precisely, if the direction $\hat{\boldsymbol{x}}$ intersects with a finite element edge on the truncating circle, and $\hat{\xi}$ is the value of the corresponding parameter, i.e.

$$
\frac{\boldsymbol{x}_{a}(\hat{\xi})}{\left|\boldsymbol{x}_{a}(\hat{\xi})\right|}=\hat{\boldsymbol{x}}
$$

the Echo Area (2.2) is evaluated by substituting in (3.28) $x=0$

$$
\left|\sum_{j, l} U_{j l} e_{j}(\hat{\xi}) \psi_{l}(0)\right|\left|\boldsymbol{x}_{a}(\hat{\xi})\right|^{\alpha} .
$$

Here, the summation extends over all IE shape functions (d.o.f.) denumerated with the double index $(j, l)$.

\subsubsection{Evaluation through postprocessing}

We shall limit our discussion to the $2 \mathrm{D}$ case. Any function $u$ that satisfies the Helmholtz equation in a domain exterior to a closed boundary $\Gamma$, along with the Sommerfeld radiation condition, satisfies automatically the Helmholtz representation formula 


$$
u(\boldsymbol{x})=\int_{\Gamma}\left\{-\frac{\partial u}{\partial n}(\boldsymbol{y}) \Phi(\boldsymbol{x}, \boldsymbol{y})+u(\boldsymbol{y}) \frac{\partial \Phi}{\partial n}(\boldsymbol{x}, \boldsymbol{y})\right\} \mathrm{d} s(\boldsymbol{y}) .
$$

Here $\boldsymbol{n}=\boldsymbol{n}(\boldsymbol{y})$ is the unit normal directed to infinity, and $\Phi$ denotes the fundamental solution to the Helmholtz operator

$$
\Phi(\boldsymbol{x}, \boldsymbol{y})=\Phi(k|\boldsymbol{x}-\boldsymbol{y}|),
$$

where

$$
\Phi(k r)=\frac{H_{0}^{(2)}(k r)}{4 \mathrm{i}} .
$$

Here $H_{0}^{(2)}$ denotes the Hankel function of the second type, of order 0 . Notice that changing the ansatz in time to $\mathrm{e}^{-\mathrm{i} \omega t}$, requires switching to the Hankel function of the first type.

Evaluating the normal derivative of the fundamental solution

$$
\frac{\partial \Phi}{\partial n}(\boldsymbol{x}, \boldsymbol{y})=k \Phi^{\prime}(k|\boldsymbol{x}-\boldsymbol{y}|) \underbrace{\boldsymbol{n}(\boldsymbol{y}) \cdot \frac{\boldsymbol{x}-\boldsymbol{y}}{|\boldsymbol{x}-\boldsymbol{y}|}}_{\cos \beta_{y}},
$$

we get

$$
u(\boldsymbol{x})=\int_{\Gamma}\left\{-\frac{\partial u}{\partial n}(\boldsymbol{y}) \Phi(|\boldsymbol{x}-\boldsymbol{y}|)+k u(\boldsymbol{y}) \Phi^{\prime}(k|\boldsymbol{x}-\boldsymbol{y}|) \cos \beta_{y}\right\} \mathrm{d} s(\boldsymbol{y}) .
$$

Using the far-field approximation

$$
|\boldsymbol{x}-\boldsymbol{y}|=|\boldsymbol{x}|-\boldsymbol{y} \cdot \hat{\boldsymbol{x}},
$$

and the asymptotic formula for the Hankel function and its derivative [1]

$$
\Phi(k r)=\mathrm{i} \sqrt{\frac{2}{\pi k r}} \mathrm{e}^{-\mathrm{i} k r}, \quad \Phi^{\prime}(k r)=\sqrt{\frac{2}{\pi k r}} \mathrm{e}^{-\mathrm{i} k r},
$$

we obtain

$$
\lim _{|\boldsymbol{x}| \rightarrow \infty}|\boldsymbol{x}|^{\frac{1}{2}}|u(\boldsymbol{x})|=\frac{1}{4} \sqrt{\frac{2}{\pi k}}\left|\int_{\Gamma}\left\{-\frac{\partial u}{\partial n}(\boldsymbol{y})+\mathrm{i} k u(\boldsymbol{y}) \boldsymbol{n} \cdot \hat{\boldsymbol{x}}\right\} \mathrm{e}^{\mathrm{i} k(\boldsymbol{y} \cdot \hat{\boldsymbol{x}})} \mathrm{d} s(\boldsymbol{y})\right| .
$$

The integration can take place over any contour surrounding the scatterer. In context of the IE discretization, it is natural to integrate over the element edges adjacent to the truncating circle.

\subsection{Evaluation of the error}

In all reported experiments, the error is computed in the $H^{1}$-semi-norm, integrated only over the nearfield domain. This is in line with treating the infinite element as an implicit implementation of an ABC only. Evaluation of the error over the whole exterior domain should be done in a weighted Sobolev norm. Since, at present, we do not understand the functional setting for the Bubnov-Galerkin case, we shall restrict ourselves to the near-field domain only and will not claim any convergence over the whole exterior domain. ${ }^{3}$ The error is reported in percent of the semi-norm of the solution, defined over the same domain. The exact solution for the cylinder problem is computed using the standard formula involving Hankel functions [14]. For the wedge problem, the unknown solution is replaced with the solution on the $h p$-refined mesh, see [11].

\footnotetext{
${ }^{3}$ We do report, however, convergence of the Echo Area.
} 


\subsection{Selection of radial shape functions: conditioning}

We do not report any extensive experiments related to conditioning. As a simple illustration supporting the idea of using integrated Jacobi polynomials, we have computed ${ }^{4}$ only the condition number for the onedimensional stiffness matrix corresponding to (3.22) and $\alpha=1$, preconditioned with the inverse of the corresponding diagonal matrix. More precisely, we have computed

$$
\operatorname{cond} \boldsymbol{B}=\frac{\lambda_{\max }^{\frac{1}{2}}\left(\boldsymbol{B} \overline{\boldsymbol{B}}^{\mathrm{T}}\right)}{\lambda_{\min }^{\frac{1}{2}}\left(\boldsymbol{B} \overline{\boldsymbol{B}}^{\mathrm{T}}\right)},
$$

where $\boldsymbol{B}=(\operatorname{diag} \boldsymbol{A})^{-1} \boldsymbol{A}$, with $\boldsymbol{A}$ denoting the complex-valued matrix corresponding to sesquilinear form (3.22), and a particular choice of shape functions. $\operatorname{diag} \boldsymbol{A}$ denotes the diagonal of matrix $\boldsymbol{A}$, and $\lambda_{\max }$ and $\lambda_{\text {min }}$ denote the largest and the smallest eigenvalues of the corresponding matrices. Three choices of radial shape functions were investigated. Along with the integrated Jacobi polynomials discussed earlier, we have also tried simple monomials, and integrated Legendre polynomials

$$
\psi_{j}(x)=\left\{\begin{array}{ll}
1, & j=0, \\
x^{j}-1, & j>1,
\end{array} \quad \psi_{j}(x)= \begin{cases}1, & j=0 \\
\int_{x}^{1} L_{j-1}(t) \mathrm{d} t, & j>1\end{cases}\right.
$$

Results presented in Fig. 2 support the idea of orthogonalizing the leading term in formula (3.22), although they are a little bit surprizing. For higher values of order $N$, the integrated Legendre polynomials perform slightly better than the integrated Jacobi polynomials. Both Legendre and Jacobi polynomials outperform significantly simple monomials. Preconditioning with the inverse of the diagonal matrix does not eliminate the effect of scaling of the shape functions. In the reported experiment, the Legendre polynomials were scaled the standard way: $L_{j}(1)=1$, and the integrated Jacobi polymials were scaled to 1 at $x=0$. An optimal scaling may result in an additional improvement in conditioning.

\subsection{Scattering of a plane wave on the rigid cylinder: verification of the code}

We begin with the standard verification test - scattering of a plane wave by a unit cylinder; for the analytical solution expressed in terms of a series of Hankel functions, see e.g. [14]. We set the wave number to $k=\pi$, and truncate the infinite domain with a circle of radius $a=3$. Fig. 3 displays convergence history for $p$-uniform and $h p$-adaptive refinements, starting with a mesh of 16 quadratic elements. The horizontal axis corresponds to the number of d.o.f. $n$ displayed on the algebraic scale $n^{1 / 3}$, with the vertical axis presenting the error on the logarithmic scale. A straight line indicates the exponential convergence

$$
\text { error } \approx C \mathrm{e}^{\beta n^{1 / 3}}
$$

predicted by the theory. Notice that the actual numbers displayed on the axes correspond to the quantities being displayed, i.e. the relative error in percent of the norm of the solution on the vertical axis, and the number of d.o.f. on the horizontal axis.

As expected, the uniform $p$ refinements deliver exponential convergence, with the adaptive refinements delivering slightly smaller error but the same rates. Fig. 4 shows the optimal $h p$ mesh, corresponding to an error of $0.025 \%$. Different colors indicate different (locally changing) order of approximation $p$ ranging from $p=1$ to $p=8$ (color scale on the right).

Fig. 5 presents contour lines of the real part of the error function, for a uniform mesh of quartic elements. The values, indicated by the color scale on the right, range from -0.006 to 0.006 . Along with the

\footnotetext{
${ }^{4}$ The computations were done by Jason Kurtz whose help we gratefully acknowledge.
} 


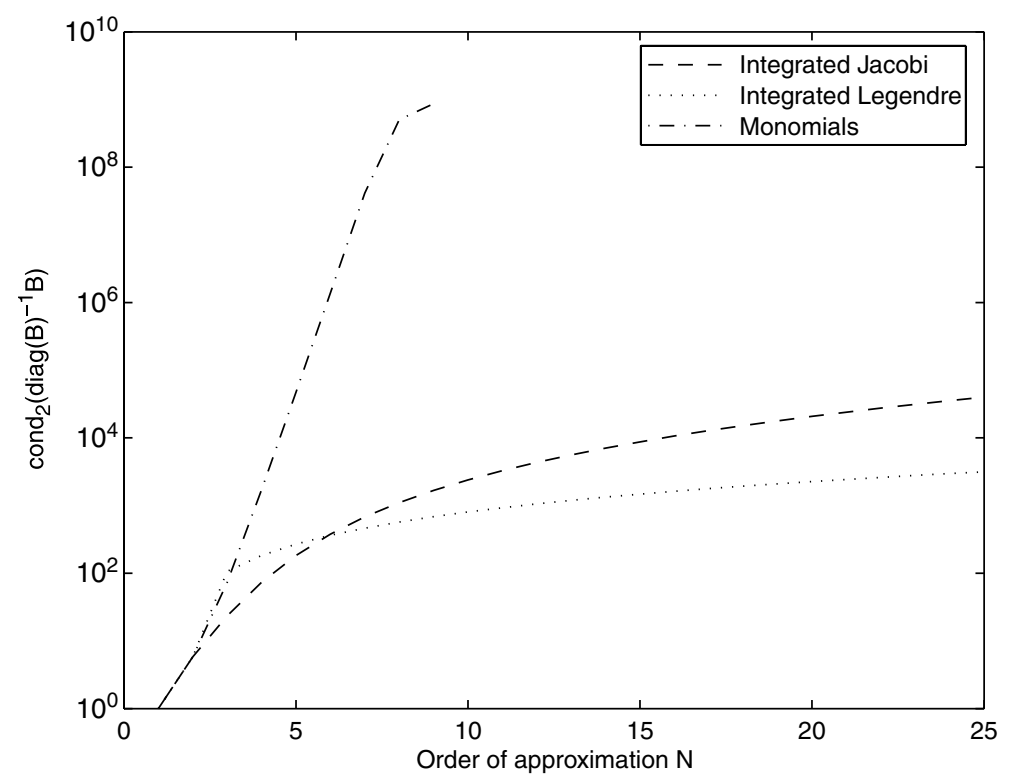

Fig. 2. Condition number (4.41) for three different choices of radial shape functions with $k a=\pi$.

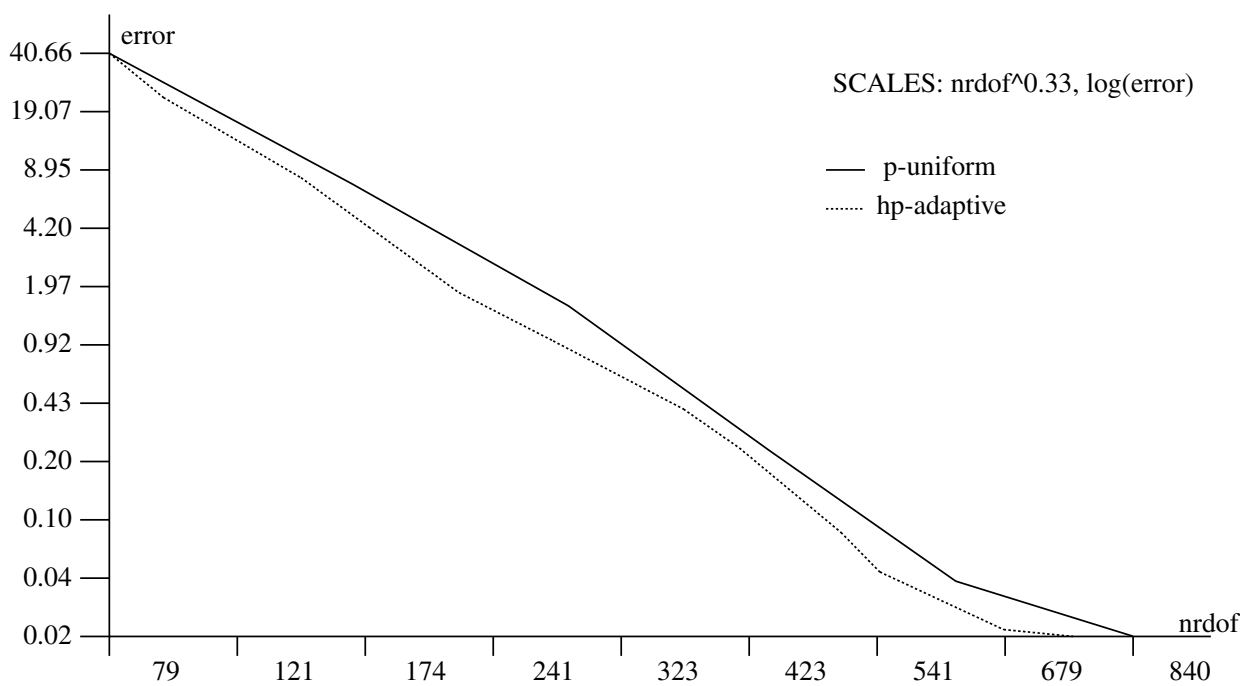

Fig. 3. Scattering of a plane wave on a cylinder. Convergence history for $p$-uniform and $h p$-adaptive refinements. The horizontal axis displays number of d.o.f. $n$ plotted on the algebraic scale $n^{1 / 3}$, and the vertical axis shows the corresponding relative error (in percent of the norm of the solution), plotted using the logarithmic scale.

FE mesh, the graph displays a portion of the infinite elements corresponding to $0.5<x<1$. The solution in the IE domain seems to be actually better than in the finite element domain which indicates that lower order infinite elements would have been sufficient. Finally, for the same mesh of quartic elements (1.5\% error), Fig. 6 presents the monostatic EA corresponding to the range of $180-0^{\circ}$ (left to right), displayed in $\mathrm{dB}$ (20 log of the actual value) versus the angle. Both methods for computing EA yield graphically indiscernible 

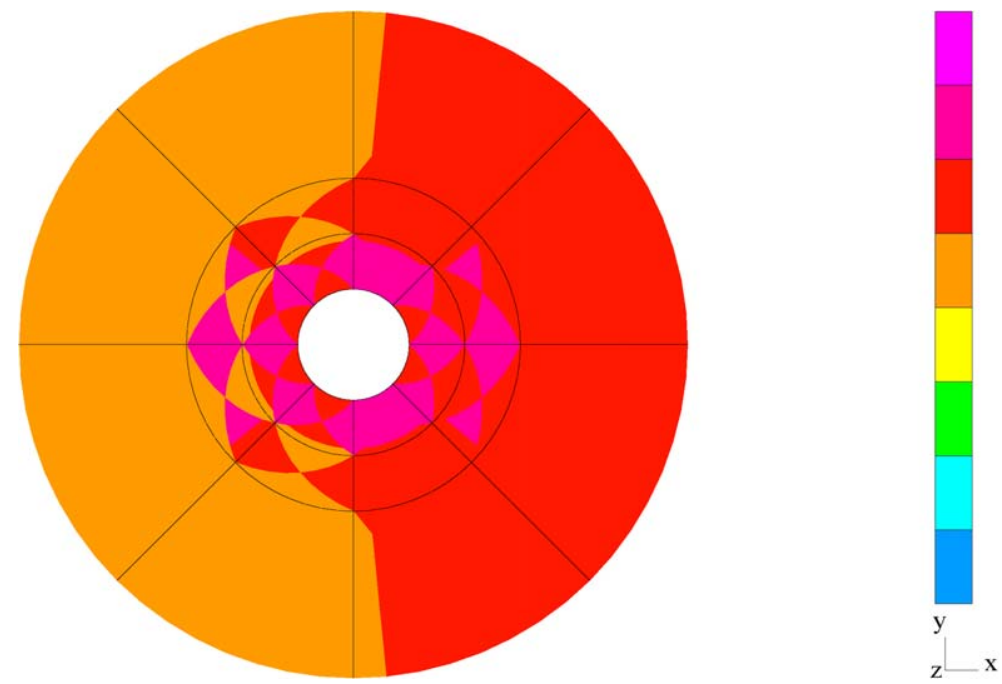

Fig. 4. Scattering of a plane wave on a cylinder. Optimal $h p$ mesh corresponding to $0.025 \%$ error. Different colors (shades of grey) indicate different (locally changing) order of approximation $p$ ranging from $p=1$ to $p=8$ (color scale on the right). (For interpretation of the references in color in this figure legend, the reader is referred to the Web version of this article.)

results (the first three significant digits are identical). The derivatives in formula (4.40) have been averaged over the adjacent finite and infinite elements. With the derivatives evaluated using only the contributions from either finite or infinite elements, the corresponding value of EA is less accurate. The slight variations of the EA for the cylinder, reflect the imperfect approximation of the geometry due to the use of isoparametric elements (for exact geometry elements, the EA would have been constant).

\subsubsection{Comparison of Bubnov- and Petrov-Galerkin formulations}

Both methods deliver practically identical results. The automatically generated $h p$ meshes are identical and the corresponding convergence graphs are visually indiscernible. Same conclusions apply to the wedge problem discussed next.

\subsection{Scattering of a plane wave on a wedge}

The second and final example deals with the resolution of singularities, and its impact on EA computations. We have divided the cylinder from the previous example into four equal quadrants and kept just one of them. We set the wave number $k=\pi$, i.e. the distance between the truncating boundary and the object is equal to one wavelength. We start with an example of a typical, automatically obtained $h p$ mesh corresponding to the incident wave coming from the right (angle $\theta=0$ ), and rather academic error level of $0.1 \%$. Figs. 7 and 8 present the optimal mesh, with three consecutive zooms showing details of the mesh around the lower corner. Fig. 9 presents convergence history for the problem using either Bubnov- and Petrov-Galerkin formulations. Similarly to the cylinder problem, the results are visually indiscernible.

\subsection{Does the resolution of singularities matter?}

We come to the final experiment reflecting the impact of adaptivity on evaluation of EA. Fig. 10 compares EA for the wedge problem evaluated using a uniform mesh of quartic elements and $h p$-adaptive meshes. The choice of the uniform meshes reflects the usual practice of selecting a mesh that reproduces 

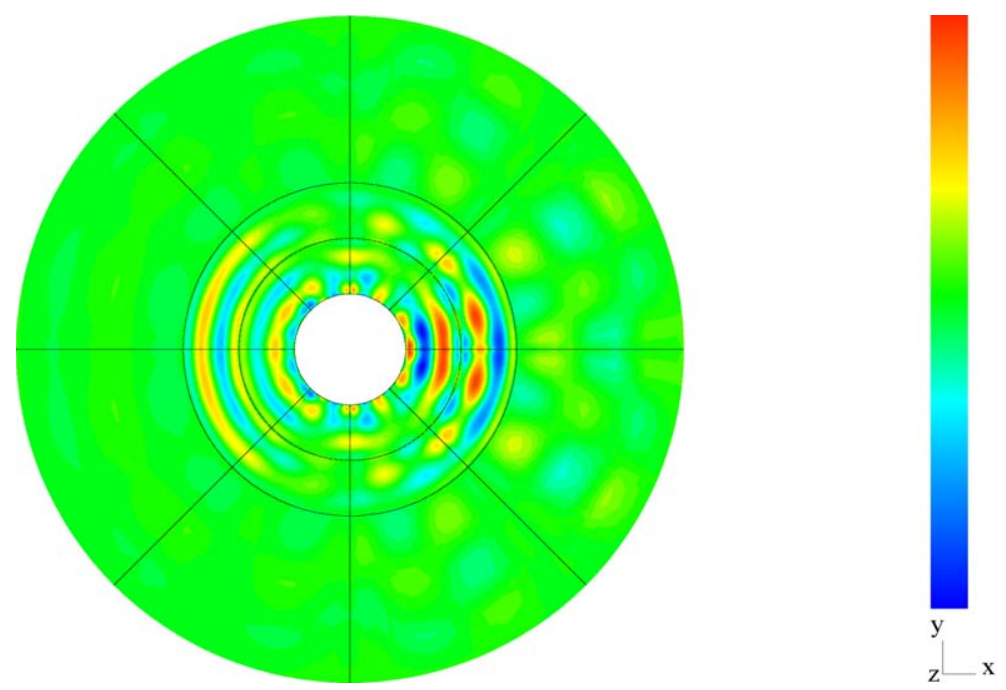

Fig. 5. Scattering of a plane wave on a cylinder. Real part of the error function for a uniform mesh of quartic elements.

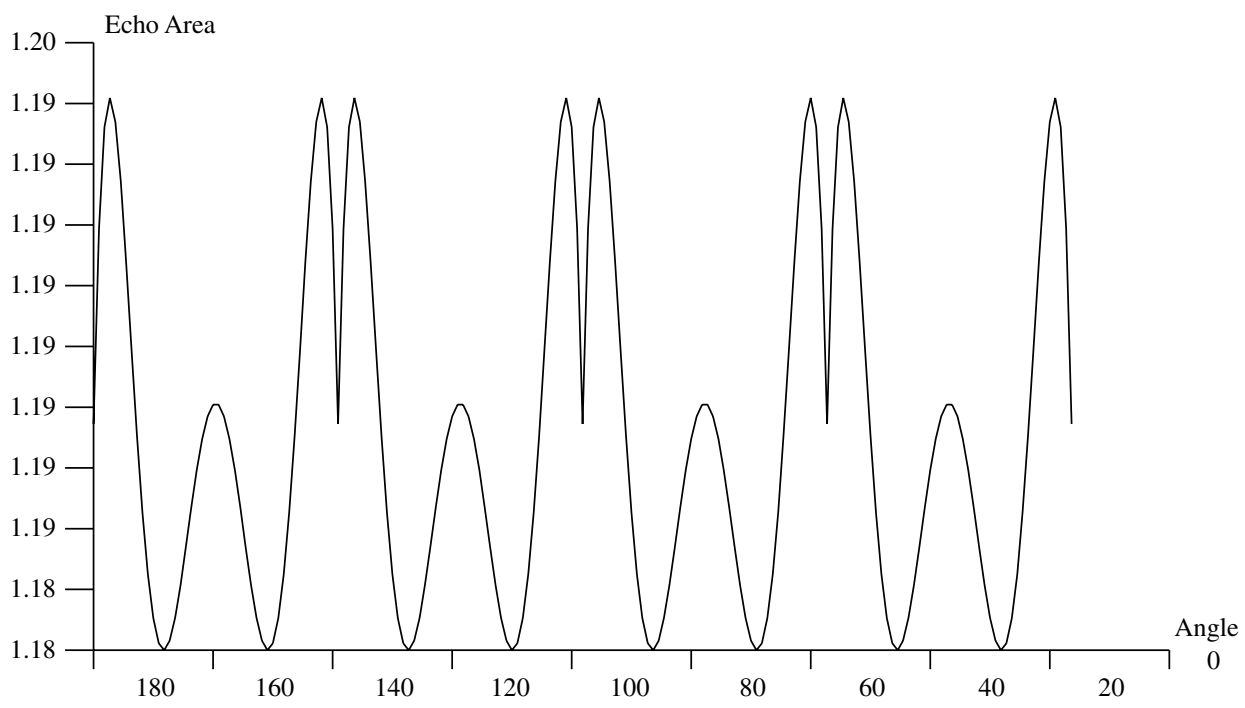

Fig. 6. Scattering of a plane wave on a cylinder. Echo Area in $\mathrm{dB}$ vs. direction of the incident wave in degrees, for a uniform mesh of quartic elements $(1.5 \%$ error $)$.

the wave form of the solution (two quartic elements per wavelength) and delivers an error in the range of $3-$ $4 \%$. The $h p$ meshes were obtained by requesting a $2 \%$ error level, at which several levels of $h p$-refinements resolve the structure of the singularities in the solution. For each direction of the incoming wave $(\theta=180,179, \ldots, 0$, left to right), the $h p$-adaptive algorithm was run starting with the optimal mesh for the previous direction, with the optimization procedure restarted from the initial mesh every $10^{\circ}$.

Except for a slight shift in the EA level, the results are practically identical. Resolution of the singularities seems to have no impact on quality of the EA computations. 

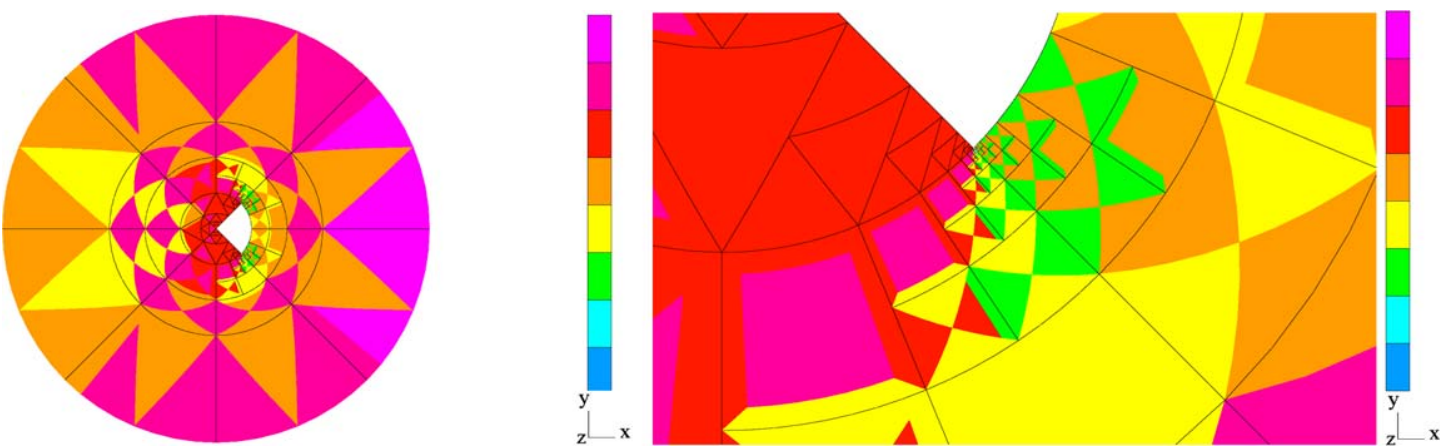

Fig. 7. Scattering of a plane wave on a wedge, $\theta=0$. Optimal $h p$ mesh for $0.1 \%$ error, with a 10 times zoom on the lower corner.

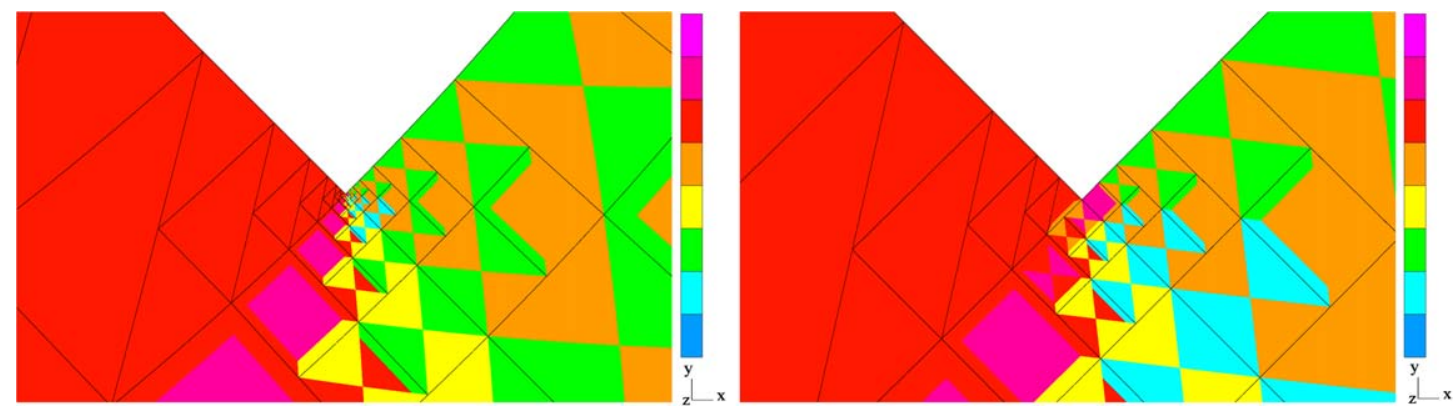

Fig. 8. Scattering of a plane wave on a wedge, $\theta=0$. Optimal $h p$ mesh for $0.1 \%$ error. Zooms on the lower corner with 100 and 1000 magnifications.

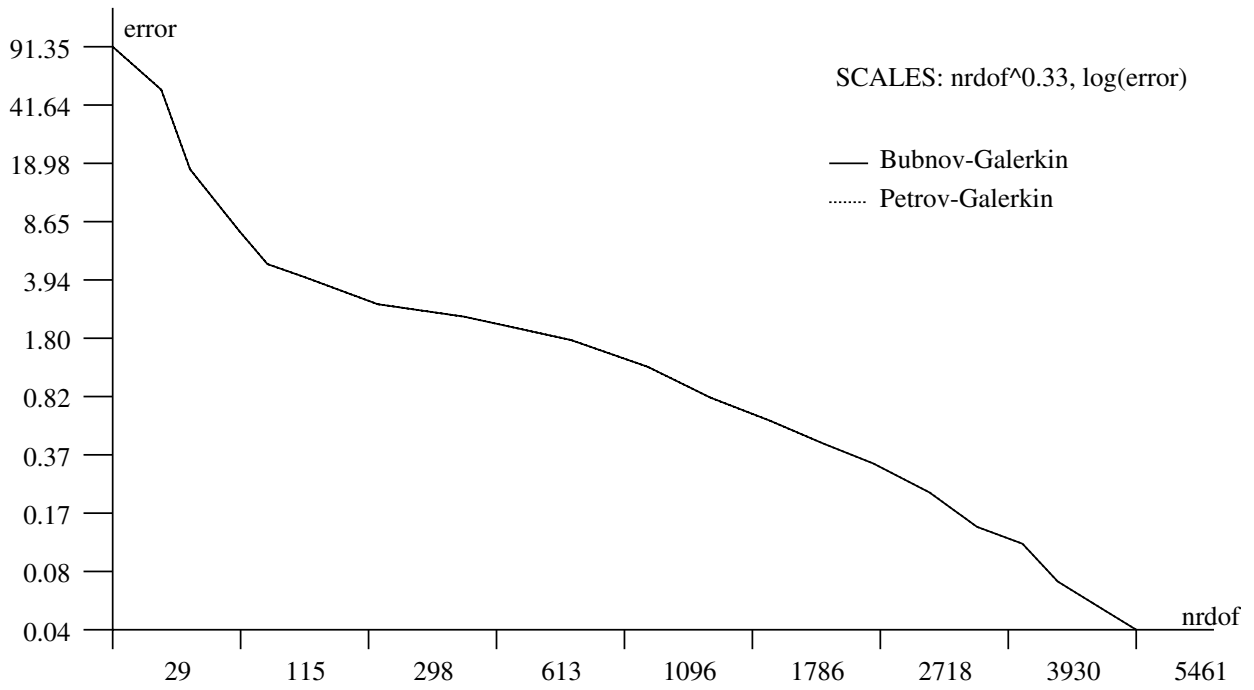

Fig. 9. Scattering of a plane wave on a wedge. Convergence history for adaptive $h p$ refinements using Bubnov-and Petrov-Galerkin IE formulations. Relative error in percent vs. the number of d.o.f. 


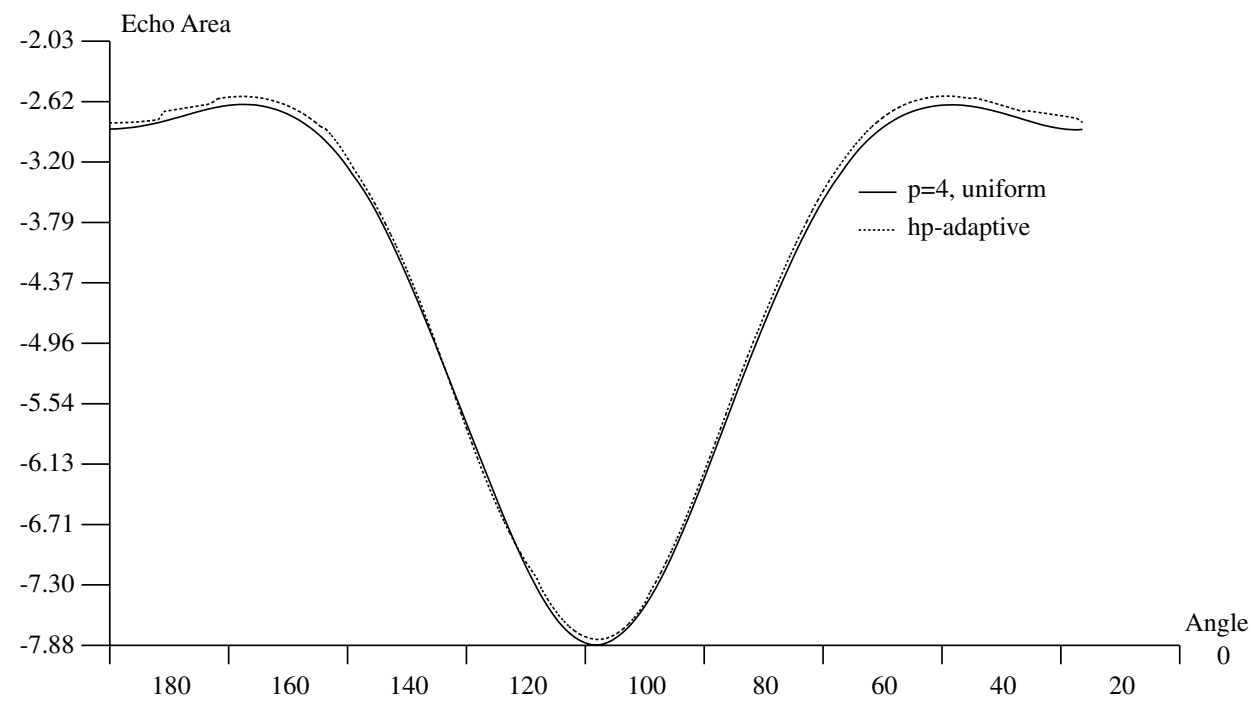

Fig. 10. Scattering of a plane wave on a wedge. Echo Area in $\mathrm{dB}$ vs. the direction of the incident wave in degrees, for the uniform mesh of quartic elements (3-4\% error range level) and $h p$-adaptive mesh ( $2 \%$ error).

We do mention, however, that for the same error level of roughly $3.5 \%$, and the quality of the corresponding EA, the adaptive algorithm delivers meshes roughly half the size of the uniform meshes. Fig. 11 presents an example of such a mesh for $\theta=90^{\circ}$. The optimal distribution of approximation order $p$ is not uniform. The fact that the algorithm has not selected any $h$-refinements, indicates that the resolution of the wave structure of the solution is more important (in terms of the energy error) than a resolution of the singularities.
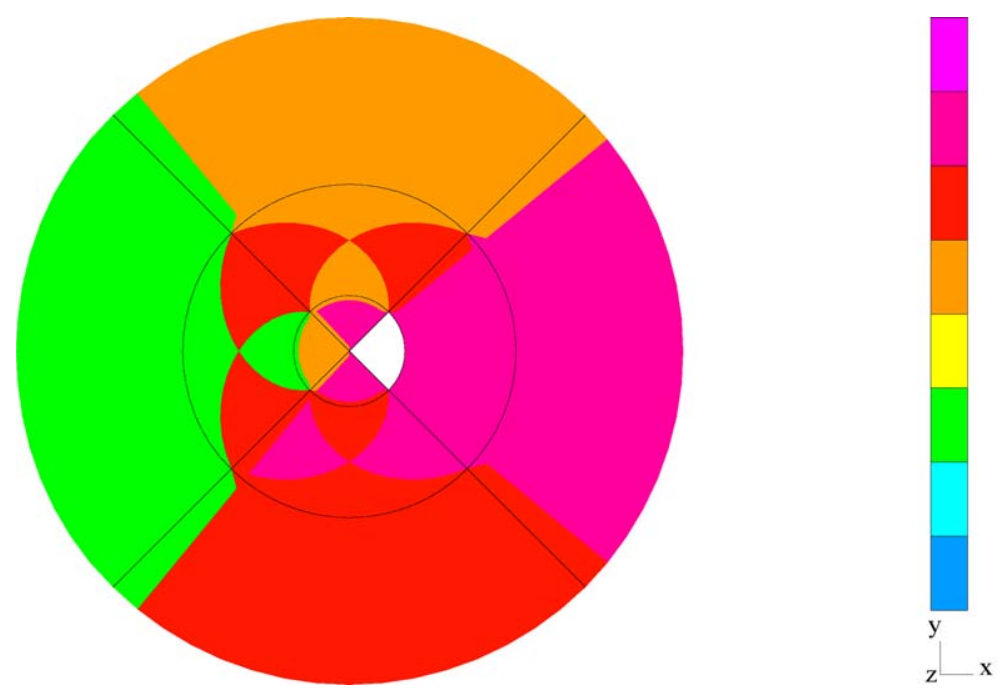

Fig. 11. Scattering of a plane wave on a wedge, $\theta=90^{\circ}$. Optimal $h p$ mesh delivering $3.5 \%$ error. 


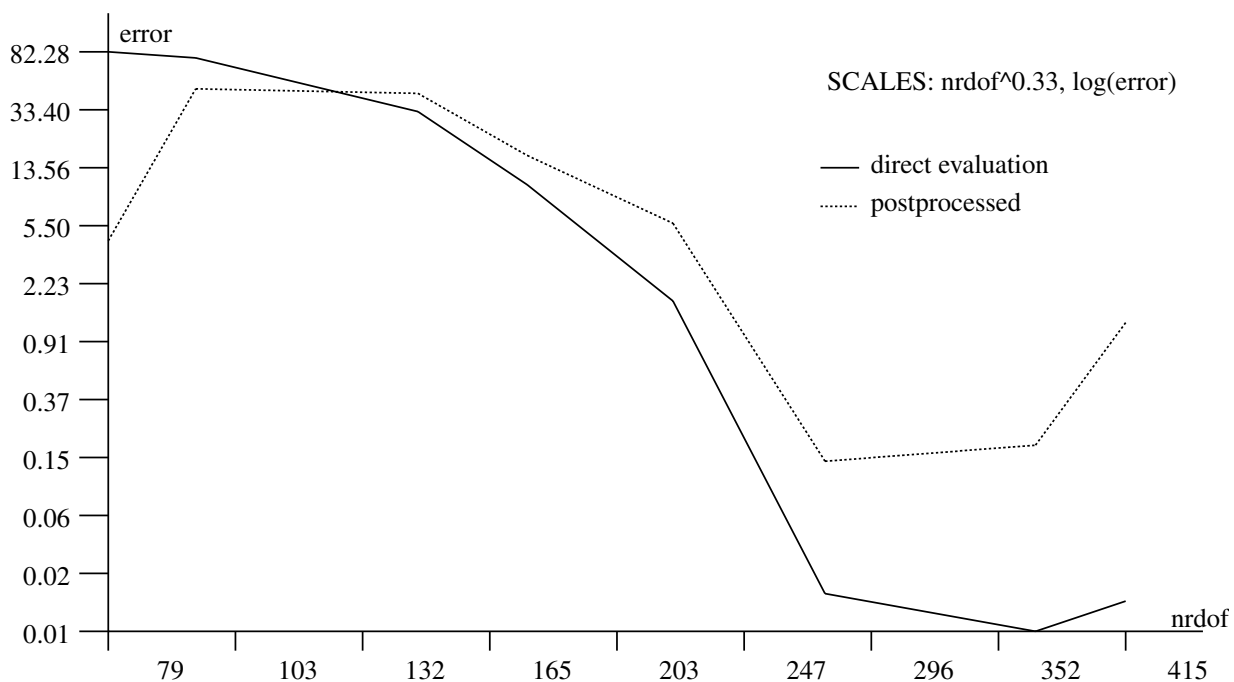

Fig. 12. Scattering of a plane wave on a cylinder. Error in EA in dB vs. the number of d.o.f.

\subsection{Evaluation of EA}

As mentioned earlier, both methods of evaluating the Echo Area, delivered practically identical results for uniform meshes with energy error level of 4-1\%. For non-uniform, adaptive meshes, the difference between the two methods is slightly more pronounced. Fig. 12 provides a more precise information showing the evolution of the error in EA for the cylinder in terms of the problem size for the sequence of optimal $h p$ meshes. The results are plotted using the algebraic and logarithmic scales, with the error reported on $\mathrm{dB}$ scale in percent of the exact value. Comparing with the energy error reported in Fig. 3, we see that indeed at the level of $2 \%$ energy error, the corresponding error in EA (in $\mathrm{dB}$ ) for both methods drops below $1 \%$ (first two digits correct) which makes again the results graphically indiscernible on the EA graph. Stable exponential convergence in terms of energy does not translate, however, into a similar exponential convergence in the quantity of interest - the Echo Area.

\section{Conclusions}

We summarize below the main conclusions of our brief considerations and numerical experiments:

1. Interpreting the integral over the exterior domain defining the sesquilinear form in the CPV sense allows the use of identical trial and test shape functions (Bubnov-Galerkin method).

2. Building the far-field pattern into the approximate solution ansatz results in almost identical formulas for the sesquilinear form in both $2 \mathrm{D}$ and 3D. The leading term, identical for the two- and three-dimensional formulations, suggests the use of integrated Jacobi polynomials for the IE shape functions.

Based on the two simple 2D numerical examples presented in this note (scattering of a plane wave on a cylinder, and on a wedge), we can make the following observations:

1. Bubnov- and Petrov-Galerkin (element of Astley et al. [3]) methods deliver essentially identical results.

2. Use of locally variable radial order in IE approximation is possible. Thus, the infinite element can be used in conjunction with the automatic $h p$-adaptivity. 
3. The Echo Area (far-field pattern) can be evaluated directly from the IE discretization without a costly postprocessing based on the Helmholtz representation formula. Again, both Bubnov- and PetrovGalerkin formulations yield identical results.

4. Resolution of singularities seems to have little impact on the quality of EA calculations.

We realize that the goal driven, and not the energy-driven adaptivity should be used to resolve efficiently the Echo Area in acoustics or Radar Cross Sections in electromagnetics (see e.g. [19]). A successful combination of goal-driven adaptivity with the automatic $h p$-adaptivity, has already been reported in $[17,15]$. The experiments on running the automatic $h p$-adaptivity algorithm for coupled FE/IE meshes reported in this note suggest that a similar strategy will pay off for the EA and RCS computations.

The $h p$ code used in the presented experiments can be downloaded from http://www.ticam.utexas.edu/ $\%$ 7Eleszek/hp-intro.html.

\section{Acknowledgement}

The work of the first author has been supported by Air Force under Contract FA9550-04-1-0050 while that of the second order is partially supported by NSF grant DMS-0311915.

\section{References}

[1] M. Abramowitz, I.A. Stegun (Eds.), Handbook of Mathematical Functions, with Formulas, Graphs and Mathematical Tables, vol. 55, National Bureau of Standards, Washington, DC (for sale by the Superintendent of Documents, US Government Printing Office, Washington, DC, 20402), 1966 (fifth printing, with corrections).

[2] R.J. Astley, J.P. Coyette, Conditioning of infinite element schemes for wave problems, Commun. Numer. Methods Engrg. 17 (2001) 31-41.

[3] R.J. Astley, G.J. Macaulay, J.P. Coyette, Mapped wave envelope elements for acoustical radiation and scattering, J. Sound Vib. 170 (1) (1994) 97-118.

[4] J.-P. Bérenger, A perfectly matched layer for the absorption of electromagnetic waves, J. Comput. Phys. 114 (1994) 185-200.

[5] P. Bettess, Infinite Elements, Penshaw Press, 1992.

[6] T.Z. Boulmezaoud, Inverted finite elements: a new method for solving elliptic problems in unbounded domains, Math. Model. Numer. Anal. 39 (1) (2005) 109-145.

[7] D.S. Burnett, A three-dimensional acoustic infinite element based on a prolate spheroidal multipole expansion, J. Acoust. Soc. Am. 96 (1994) 2798-2816.

[8] L. Cremers, K.R. Fyfe, J.P. Coyette, A variable order infinite acoustic wave envelope element, J. Sound Vib. 17 (4) (1994) $483-508$.

[9] L. Demkowicz, 2D $h p$-adaptive finite element package (2Dhp90), version 2.0, Technical Report 06, TICAM, 2002.

[10] L. Demkowicz, F. Ihlenburg, Analysis of a coupled finite-infinite element method for exterior Helmholtz problems, Numer. Math. 88 (2001) 43-73.

[11] L. Demkowicz, W. Rachowicz, Ph. Devloo, A fully automatic $h p$-adaptivity, J. Sci. Comput. 17 (1-3) (2002) $127-155$.

[12] K. Gerdes, L. Demkowicz, Solution of 3D Laplace and Helmholtz equation in exterior domains using $h p$ infinite elements, Comput. Methods Appl. Mech. Engrg. 137 (1996) 239-274.

[13] D. Givoli, Numerical Methods for Problems in Infinite Domains, Elsevier, Amsterdam, 1992.

[14] M.C. Junger, D. Feit, Sound, Structures, and their Interaction, MIT Press, Boston, 1986.

[15] D. Pardo, L. Demkowicz, C. Torres-Verdin, L. Tabarovsky, A goal-oriented $h p$-adaptive finite element method with electromagnetic applications. Part I: Electrostatics, Technical Report 57, ICES, 2004.

[16] A. Safjan, M. Newman, On two-dimensional infinite elements utilizing basis functions with compact support, Comput. Methods Appl. Mech. Engrg. 190 (48) (2001) 6399-6424.

[17] P. Solin, L. Demkowicz, Goal-oriented $h p$-adaptivity for elliptic problems, Comput. Methods Appl. Mech. Engrg. 193 (2004) 44 468.

[18] S.V. Tsynkov, Numerical solution of problems on unbounded domains. a review, Appl. Numer. Math. 27 (1998) $465-532$.

[19] A. Zdunek, W. Rachowicz, A three-dimensional $h p$-adaptive finite element approach to radar scattering problems, in: Fifth World Congress on Computational Mechanics, Vienna, Austria, 2002. 